\title{
Jean Taricat, Suburbia. Une utopie libérale
}

Paris, Éditions de la Villette, 2013, 157 p., 19 euros.

\section{Bénédicte Grosjean}

\section{(2) OpenEdition \\ Journals}

Édition électronique

URL : http://journals.openedition.org/crau/435

DOI : $10.4000 /$ crau. 435

ISSN : 2547-5746

Éditeur

Éditions du patrimoine

Édition imprimée

Date de publication : 1 décembre 2014

Pagination : 259

ISBN : 978-2-7577-0379-3

ISSN : 1296-4077

Référence électronique

Bénédicte Grosjean, « Jean Taricat, Suburbia. Une utopie libérale », Les Cahiers de la recherche

architecturale et urbaine [En ligne], 30/31 | 2014, mis en ligne le 14 septembre 2017, consulté le 24

septembre 2020. URL : http://journals.openedition.org/crau/435 ; DOI : https://doi.org/10.4000/crau. 435 


\section{Jean Taricat, Suburbia. Une utopie libérale}

Paris, Éditions de la Villette, 2013, 157 p., 19 euros.

À la manière des Congrès internationaux d'architecture moderne (CIAM), qui ont commencé par la question du Logement minimum en 1929 pour aboutir à celle de la Ville fonctionnelle en 1933, les travaux de Jean Taricat forment à première vue une montée dans les échelles: avec son histoire du Logement à bon marché, publié dans les années 1980 et aujourd'hui épuisé ; puis le livre Histoires d'architecture (2004), qui partait des objets construits pour remonter vers leurs projets, parfois implicites ; et cette troisième publication, qui propose une relecture de l'histoire de l'urbanisme à travers un point de vue à nouveau décalé : la formation progressive de la "suburbia ". Face à la pléthore d'analyses ou de pamphlets sur ce phénomène, à côté des nombreuses approches économiques, géographiques ou anthropologiques, on perçoit d'emblée la complémentarité assez fructueuse de cet ouvrage, avec par exemple les travaux de David Mangin, également aux Éditions de la Villette (La Ville franchisée, 2004). Celui-ci avait en effet abordé, sur des territoires contemporains banals et au moyen des petits croquis au feutre aujourd'hui célébres, plusieurs des thèmes importants développés ici : la ville sectorisée par la hiérarchisation des voies; la ville de l'entre-soi ; la ville qui se privatise.

Avec cet ouvrage, Jean Taricat apporte une connaissance historique des théories sous-jacentes à ces grandes tendances, éclairée par un riche corpus iconographique, d'archives, de plans, revues et photographies d'époque, dont le mode de reproduction de cette édition, monochrome, ne rend peut-être pas assez la grande qualité. II illustre ainsi la variété de formalisations de la "suburbia » par un nombre important d'opérations de référence, certaines souvent croisées dans les travaux d'architectes mais parfois instrumentalisées à force d'être décontextualisées. L'auteur en replace ici tous les enjeux dans un faisceau de doctrines et d'idéologies sociales et politiques.

Car s'il est spécialisé dans I'histoire de I'architecture, Jean Taricat est sociologue de formation et professeur de métier : il enseigne cette discipline depuis une trentaine d'années, à Paris-Tolbiac d'abord puis à l'école d'architecture, de la ville et des territoires de Marne-la-Vallée, dont il a participé à la fondation dans les années 1990 et qu'il a dirigée de 2002 à 2005. Ce livre, qui vient étoffer la collection de publications issues de cette école chez le même éditeur (avec la revue Marnes et Où va la ville aujourd'hui, de Jacques Lucan), témoigne ainsi de savoirs longuement approfondis, offrant plusieurs niveaux de lecture, mais aussi d'un savoir- 
faire, tant la clarté et la fluidité d'un cours maintes fois retravaillé s'y ressent.

\section{Une histoire de l'urbanisme assez complète sur le $x x^{e}$ siècle}

$\grave{A}$ un premier niveau, cet ouvrage brosse I'histoire de la manière dont on a pensé, depuis les années 1850, les formes d'implantation d'une nouvelle population, d'abord à la campagne, puis comment celles-ci ont été «le laboratoire des formes urbaines de la métropole contemporaine » (p. 11), ce qui ne constitue pas la moindre des hypothèses développées dans la première et plus importante partie ("Des formes suburbaines »). Aussi ne se lit-il pas seulement comme l'histoire de la suburbia au sens d'un territoire périphérique, d'une morphologie peu dense ou de la propriété individuelle. Par l'analyse des relais d'influences et de diffusion des théories, l'ouvrage devient une histoire de l'urbanisme assez complète sur le $x x^{e}$ siècle, englobant par exemple, outre les opérations de banlieue et autres périphéries lointaines, les squares en plein Londres du XIX siècle, le plan de Patrick Geddes pour Tel-Aviv (1925), la problématique du " cœur de la ville » débattue par les CIAM dans les années 1950, ou encore les villes nouvelles compactes, les logements collectifs sur dalle, les années 1960 en Angleterre. La loi Loucheur (1928), en revanche, bien que productrice de centaines de kilomètres carrés d'habitat pavillonnaire, n'occupe que deux paragraphes de cette histoire qui se centre sur l'urbanisme dessiné, planifié, et sur les théories qui le charpentent.

\section{La périurbanisation comme problème}

Mais si le périurbain n'est pas le seul objet analysé dans le livre, la périurbanisation est bien le principal problème qui occupe l'auteur, comme il en ressort de la seconde partie ("Une utopie libérale »). Deux fois plus courte, elle est sous-tendue par un doute récurrent, "existe-il aujourd'hui une possibilité d'enrayer le sprawl ?» (p. 125), et documentée par un autre type de corpus : des cartes et statistiques de I'Insee, des enquêtes de ménages, des études de I'laurif et d'autres spécialistes de l'immobilier. Remplaçant le qualificatif de Bourgeois utopias proposé par Robert Fishman (The Rise and Fall of Suburbia 1987), par celui « d'utopie libérale », Jean Taricat revient aux sources de ce mouvement, tant chez John Locke que chez Alexis de Toqueville, montrant d'un côté que toutes les couches de la société sont concernées et, de l'autre, que le projet libéral est aussi progressiste, en tout cas dans un contexte principalement anglosaxon, de Ebenezer Howard aux auteurs contemporains comme Peter Saunders. La notion de société civile, par exemple, y a une connotation beaucoup plus positive qu'en France, et on y voit son émergence d'un bon œil, comme signe de bonne santé démocratique. Du côté français du Channel, cette deuxième partie éclaire de manière détaillée les liens entre le milieu périurbain et la prépondérance des propriétaires occupants, le caractère assez illusoire d'un "retour au centre ", le rôle crucial de ce processus dans le développement économique de I'après-guerre, ou encore, les ambiguïtés de la posture de l'État. Celui-ci a, en effet, à la fois investi massivement dans les villes nouvelles et soutenu les politiques d'aide à la personne, rendant solvables pour l'immobilier une grande quantité de ménages « captifs des HLM » (p. 126).

\section{Entre deux excès, de planification et de laisser-faire}

La première partie peut alors se relire sous un angle plus thématique que chronologique, pour y suivre la formation de la suburbia à travers deux grands mouvements : I'un, progressif, la démocratisation du rêve de la propriété individuelle (chapitre I, "L'Arcadie pour tous ») ; l'autre, dialectique, une oscillation entre deux types de rejet : celle d'une planification d'échelle massive et générique et celle du "laisser-faire », fragmentant et chaotique (chapitre ॥, "Sprawl !», et chapitre III, "Planifier le sprawl »).

L'histoire démarre avec les villas de type palladien construites dans les campagnes de la Vénétie - curieusement devenues aujourd'hui le paradigme de la «ville diffuse " - pour de riches marchands qui s'y ressourçaient au contact de la nature et de l'agriculture, s'inspirant en cela, selon James Ackerman, des grecs de l'Arcadie légendaire. Si ces séjours 
étaient encore longs et espacés, Taricat date du début du XIX siècle le moment où, pour une population très aisée, il devient possible d'habiter à la campagne et "d'avoir ses affaires" en ville. Plus d'un siècle de lotissements, au sens large, est alors parcouru dans ce chapitre, depuis le premier hameau par John Nash de huit cottages au fond de la propriété d'un banquier quaker (Blaise Hamlet, Bristol, 1810 ca.), jusqu'aux soixante maisons à patio de Jørn Utzon (Kingo Housing, Copenhague, 1953) également disposées en grappe autour d'un parc commun, en passant par les grands classiques, Riverside, Le Vésinet, Hampstead Garden Suburb et Radburn.

Mais I'intérêt spécifique de ce travail réside dans les fils thématiques qu'il tisse entre les opérations (qu'on aurait peut-être pu doter de quelques données comparatives systématiques, comme le nombre de logements à l'hectare ou une échelle graphique sur les plans) : la diminution de la taille de la parcelle avec la démocratisation de la propriété ; la définition progressive des dimensions de "l'unité de voisinage "; la différenciation des voies de circulation, principales et secondaires, voiture et piétonne, rocades et pénétrantes, qui mène des closes de Unwin au rapport Buchanan (1963).

Le deuxième chapitre traite sous un titre claquant, "sprawl !", de changements d'échelle colossaux, concernant la seule décennie 1950 : celui des nappes pavillonnaires, avec des pages instruc- tives sur le phénomène des Levittowns aux États-Unis, qui illustrent à la fois la propriétarisation de la classe moyenne, I'industrialisation de la production et la privatisation de l'urbanisme; celui de la décentralisation qui devient industrielle, celui de l'enclavement qui produit la spécialisation et la ségrégation, celui du commerce avec l'émergence des shopping centers. La manière dont Victor Gruen associait la production de ces espaces à une véritable préoccupation sur "le cœur des villes" amène, dans le troisième chapitre sur les années 1960 à une réflexion très documentée sur la recherche de densification de la banlieue à travers la politique des villes nouvelles, soulignant à quel point l'habitat est $y$ aussi en grande majorité individuel, voire pavillonnaire. Importante également est la réflexion sur la résurgence dans ce contexte de la notion de "village», analysée de manière détaillée en comparant ses formes et occurrences, chez les partisans du Townscape comme chez les jeunes modernistes du Team $X$

Le fil de la réflexion sur la suburbia aurait peut-être pu passer par l'Italie de ces années-là, où s'élaborait la notion de «ville-territoire» (Manfredo Tafuri), se reprenait celle de "ville-région» (Giancarlo De Carlo), prémisses de la «ville diffuse ». En effet, celle-ci ne représente pas seulement une forme d'urbanisation dispersée, telle qu'elle est effectivement abordée au chapitre suivant. C'est auss une théorie pour penser l'urbanisation contemporaine et, en particulier, l'individualisation des choix, les micromutations parcellaires, les formes de l'entre-soi (de la famille élargie, dans le Veneto, à la clubbisation formulée par Éric Charmes), toutes thématiques devenant cruciales dans la suite du texte.

\section{Comprendre les acteurs individuels}

Sous les analyses déjà évoquées, on décèle en effet dans la dernière partie le souci de l'auteur de comprendre les acteurs individuels, les habitants : "On peine à croire que les seize millions de Français recensés récemment dans l'espace périurbain aient été victimes d'une illusion collective " (p. 69). L'histoire du suburbain, c'est l'histoire de l'urbanisme, $c^{\prime}$ est la nôtre. Et on finit par se demander ce qui pose tellement question : Est-ce la propriété individuelle ou l'initiative individuelle qui est en jeu ? Est-ce l'absence de densité ou le chaos apparent du « laisserfaire " ? Est-ce qu'on dénonce les préférences de la majorité de la société, ou le fait qu'elles soient politiquement acceptées, soutenues?

À cela, l'auteur apporte finalement deux points de vue qui déplacent pertinemment les critères habituels. II montre qu'au-delà du gain d'espace et même du rapport à la nature, c'est le potentiel d'évolution, le droit à la transformation de cet habitat qui est plus que tout recherché, ouvrant ainsi la perspective vers de nouveaux champs projectuels. À l'inverse, la consommation d'espace et sa 
sectorisation l'inquiète bien moins que la prise de pouvoir dans les communes par une forme de société civile, devenue conservatrice avec le patrimoine acquis, mettant là le doigt sur d'autres paradoxes, ceux de la démocratie participative.

BÉNÉDICTE GROSJEAN

Ingénieur civil architecte et docteur en urbanisme, chercheur au LACTH, maître assistant à l'Ensap de Lille, domaine

"Ville et territoire», chargée de cours à la faculté d'architecture, d'ingénierie architecturale et d'urbanisme ( $\mathrm{LOCl}$, site de Tournai) de I'UCLouvain. 